\title{
Nierenschonende blutzuckersenkende Therapie
}

\begin{abstract}
Nach und nach werden immer mehr Daten aus der Studie EMPA-REG OUT$\mathrm{COME}^{\oplus}$ präsentiert, u.a. zu den renalen Endpunkten der Studie. Prof. Christoph Wanner, Würzburg, präsentierte sie in New Orleans. Ein präspezifizierter sekundärer Endpunkt war die Manifestation oder Verschlechterung einer Nephropathie, definiert als Progression zur Makroalbuminurie $>300 \mathrm{mg} / \mathrm{g}$, Verdoppelung des Serumkreatinins (begleitet von einem eGFR-Abfall $\leq 45 \mathrm{ml} /$ $\min / 1,73 \mathrm{~m}^{2}$ ), Beginn einer Nierenersatztherapie oder aber Tod durch Niereninsuffizienz.
\end{abstract}

Die eGFR lag zu Beginn im Mittel bei etwa $74 \mathrm{ml} / \mathrm{min} / 1,73 \mathrm{~m}^{2}$, ein Viertel der Teilnehmer hatte bereits eine Nierenin- suffizienz (eGFR $\left.<60 \mathrm{ml} / \mathrm{min} / 1,73 \mathrm{~m}^{2}\right)$, etwa $40 \%$ eine Albuminurie. Die Kontrolle erfolgten alle drei Monate.

Die Risikoreduktion für die genannten Nierenereignisse innerhalb der Beobachtungszeit von drei Jahren lag mit Empagliflozin vs. Placebo bei 39\% ( $\mathrm{p}<$ 0,001). Die Dosierung des Gliflozins machte keinen Unterschied. Bei jenen, die schon zu Studienbeginn niereninsuffizient waren, wurde das Risiko einer weiteren Verschlechterung durch das Gliflozin um $42 \%$ gesenkt $(\mathrm{p}<0,001)$.

\section{Glomerulärer Hochdruck sinkt}

Während sich die Nierenfunktion in der Placebogruppe kontinuierlich verschlechterte, blieb sie in den beiden Ve- rumgruppen nach initial leichtem eGFR-Abfall weitgehend stabil. Wanner erklärte diesen Verlauf mit dem Wirkmechanismus von Empagliflozin. Die SGLT-2-Hemmung stelle wahrscheinlich das glomeruläre Feedback wieder her, indem zusammen mit Glucose weniger rückresorbiertes Natrium an der Makula densa die Angiotensinsekretion forciert. Die daraufhin erfolgende Engstellung des Vas afferens am Glomerulum reduziert den bei Diabetikern erhöhten intraglomerulären Druck und damit die Filtration.

Sarah Louise Pampel

- 76th Scientific Sessions der American Diabetes Association: "Update from the EMPA-REG OUTCOME Trial"; New Orleans, Juni 2016

\section{Spannungskopfschmerzen}

\section{0\%iges Pfefferminzöl in der Akuttherapie bestätigt}

— In einer neuen, apothekengestützten, nicht-interventionellen Studie wurde erneut die natürliche Behandlung von Kopfschmerzen vom Spannungstyp mit einer 10\%igen Pfefferminzöllösung (Prüfpräparat: Euminz ${ }^{\circledR}$ überprüft: Mehr als $80 \%$ der 271 Studienteilnehmer beurteilten die Wirksamkeit und über 97\% die Verträglichkeit des Prüfpräparats als sehr gut oder gut. Bereits am ersten Tag verschwanden die Kopfschmerzen bei $43 \%$ vollständig. Bei weiteren $44 \%$ wurden die Beschwerden deutlich reduziert.

\section{Schmerzmitteleinnahme sinkt}

Das besondere Plus: 75\% der Teilnehmer konnten durch die Verwendnung des Pfefferminzöls die Einnahme eines systemischen Analgetikums vermeiden. Das Fazit von Dr. Ronald Brand, Königstein: „Die Schmerzlinderung bei leich- ten bis mittleren Kopfschmerzen und die Dauer bis zum Wirkungseintritt ist mit herkömmlichen, systemischen Schmerzmitteln vergleichbar - die Linderung der Schmerzen ist nach 15-30 Minuten zu spüren.“ Das Pfefferminzöl aktiviert analgetische Wirkmechanismen sowohl auf zentraler als auch peripherer Ebene.

\section{Einziges lokal anzuwendendes Arzneimittel}

Euminz $z^{\oplus}$ ist in Deutschland als einziges lokal anzuwendendes natürliches Arzneimittel zur Behandlung von leichten bis mittelschweren Kopfschmerzen vom Spannungstyp zugelassen. Auch für Kinder ist das topische Pfefferminzöl verordnungs- und erstattungsfähig. $\mathrm{Zu}-$ dem wurde 10\%-ige Pfefferminzöllösung (nur in Euminz ${ }^{\circledast}$ enthalten) aktu-

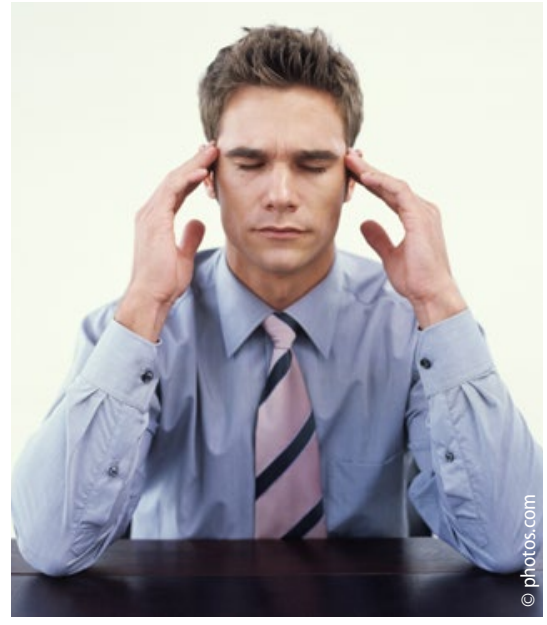

Spannungskopfschmerzen lassen sich auch lokal behandeln.

ell in die neue Praxis-Leitlinie zu primären Kopfschmerzerkrankungen der Deutschen Gesellschaft für Schmerzmedizin (DGS) aufgenommen.

Red.

- Nach Informationen von Klosterfrau 